\title{
Cenários epidemiológicos da pandemia de SARS-CoV-2
}

\author{
Nascimento, J. O. do ${ }^{2^{*}} ;$ Passos, C. A. C. ${ }^{1^{*}} ;$ Moret, M. A A $^{2,3}$ \\ 1 Departamento de Física, Universidade Federal do Espírito Santo, UFES, Vitória/ES, Brasil. \\ 2 Departamento de Modelagem Computacional, CIMATEC, Salvador/BA, Brasil. \\ 3 Universidade do Estado da Bahia, UNEB, Salvador/BA, Brasil. \\ *e-mail: carlos.passos@ufes.br, jeffersonascimento@gmail.com
}

\begin{abstract}
Resumo
A pandemia de Coronavírus se apresentou como um dos problemas mais complexos de saúde pública, em sua magnitude, até o presente momento da história da humanidade. Uma possibilidade de orientar as políticas públicas para combater esta enfermidade é a proposição de modelos preditivos em relação a difusão do SARS-CoV-2. Este artigo tem como objetivo apresentar alguns métodos, ajustes não-lineares e modelos computacionais utilizados para realizar estimativas sobre a propagação do Coronavírus em países como a Itália e Brasil. Destaca-se a proposição de um Modelo de Física do Estado Sólido como método de previsão. Uma curva sigmoide especificamente utilizada em processos de transformação de materiais sólidos: a equação de Avrami ou de Johnson-Mehl-Avrami-Kolmogorov (JMAK). Destacam-se também o modelo SEIAHR e os ajustes não-lineares por meio de Gaussianas. Ao fim, podemos inferir que as metodologias utilizadas foram capazes de propor um padrão quanto ao processo de disseminação do Coronavírus acerca das regiões estudadas.
\end{abstract}

Palavras-chave: Cenários epidemiológicos, Coronavírus, Covid-19

\begin{abstract}
The Coronavirus pandemic has presented itself as one of the most complex public health problems, in its magnitude, until the present moment in human history. One possibility to guide public policies to combat this disease is the proposal of predictive models in relation to the spread of SARS-CoV-2. This article aims to present some methods, nonlinear adjustments and computational models used to make estimates about the spread of Coronavirus in countries like Italy and Brazil. The proposal of a Solid State Physics Model as a forecasting method stands out. A sigmoid curve specifically used in solid material transformation processes: the Avrami or Johnson-Mehl-Avrami-Kolmogorov (JMAK) equation. Also noteworthy are the SEIAHR model and non-linear adjustments using Gaussians. In the end, we can infer that the methodologies used were able to propose a standard regarding the process of spreading the Coronavirus in the studied regions.
\end{abstract}

Keywords: Epidemiological scenarios, Coronavirus, Covid-19

\section{Introdução}

A pandemia causada pelo vírus SARS-CoV-2, agente etiológico da Covid-19 (i.e. Conronavirus disease), representa uma ameaça de magnitude não enfrentada neste século. Por causa disto, cada governo propôs medidas de saúde pública fundamentais para se retardar a transmissão e a propagação do vírus e mitigar seus impactos. Especificamente o surto no Brasil, desencadeou um grande conjunto de casos infectados por Covid-19.
Uma vez que não há medicamentos ou vacinas comprovadamente para o tratamento da doença, é um muito importante fazer análise dos dados de casos de infecção e a sua interpretação matemática para fundamentar as medidas governamentais para a supressão e mitigação do impacto da Covid-19. Isto significa que é necessário fazer estimativas através de modelos matemáticos para avaliar o potencial de desenvolvimento de transmissão humano-humano sustentado. Uma vez que a doença apresenta suas próprias características biológicas, os modelos precisam ser adaptados a variabilidade das 
características das regiões, das tomadas de decisões governamentais e da população, a fim de serem capazes de lidar com situações reais. Assim, neste artigo, temos a intenção de colaborar com a compreensão do atual quadro epidemiológico através do estudo de um modelo matemático por equações diferenciais ordinárias e modelo similares que possam descrever a propagação da doença infecciosa de transmissão pessoa a pessoa.

Nosso intuito de seguir neste viés, ocorre pela necessidade em estimativas em relação a pandemia de Covid-19, pois, os números reais dependem totalmente da testagem em massa da população [1]. Algo que não ocorreu no Brasil em 2020 e em muitos países. Por exemplo, em estudos recentes sobre a pandemia surgem trabalhos científicos que sugerem para 0 Estado do Pará a multiplicação dos números oficiais por valores entre 7-20. [1]

Diante do exposto, o objetivo da presente pesquisa é apresentar cenários epidemiológicos à difusão do Coronávírus. Utilizaremos alguns métodos tradicionais em Epidemiologia por meio da modelagem computacional, ajustes não-lineares e o

Modelo da equação de Avrami ou de Johnson-MehlAvrami-Kolmogorov (JMAK), que apresentou resultados robustos para tal propagação viral.

\section{Metodologia}

Para que pudéssemos realizar as análises propostas neste artigo, a base de dados oficial utilizada é a fornecida pelo Ministério da Saúde. Para realizarmos análises preditivas em relação à difusão do Coronavírus, apresentaremos alguns métodos que utilizaremos nesta pesquisa.

\subsection{Modelo SIR}

Este modelo epidemiológico foi proposto por Kermack e McKendrick [2], leva em consideração as seguintes variáveis:

1. Indivíduos susceptíveis que ainda não foram contaminados pela doença. $\mathrm{S}(\mathrm{t})$ é usado para representar o número de indivíduos não infectados com a doença no momento $t$, ou aqueles suscetíveis à doença.

2. Indivíduos infectados que são aqueles que tiveram contato com a doença (contaminados). I(t) representa o número de indivíduos que tenham sido infectadas com a doença e que são capazes de transmitir a doença aos da categoria susceptível.

3. Indivíduos removidos que são representados por aqueles recuperados (curados) e mortos. $R(t)$ é o compartimento utilizado para aqueles indivíduos que foram infectados e, em seguida, removidos a partir da doença, quer devido à imunização ou devido à morte. Vamos considerar que nesta categoria as pessoas curadas não são capazes de serem infectados novamente ou para transmitir (numa janela de tempo) a infecção a outras pessoas. O fluxo do presente modelo pode ser considerado da seguinte forma:

$$
S \rightarrow I \rightarrow R
$$

Levamos em consideração que o número de indivíduos em cada uma das três classes muda em relação ao tempo, isto é, $S(t), I(t)$ e $R(t)$ são funções dependentes do tempo $t$. Portanto, o total da população é a soma do número de indivíduos que estão em cada uma das classes em qualquer tempo $t: \mathrm{N}=S(t)+I(t)+$ $R(t)$. Isto simplifica o modelo.

Kermack e McKendrick [2] apresentam um modelo matemático cuja distribuição estatística segue uma normal, que denota os casos que são removidos por morte ou recuperação em uma epidemia:

$$
\frac{d z}{d t}=\frac{l^{3}}{2 x_{0} k^{2}} \sqrt{-q} \sec h^{2}\left(\frac{\sqrt{-q}}{2} l t-\phi\right)
$$

Conforme Savi, Savi e Borges [3] O modelo SIR busca resolver o sistema de equações diferenciais ordinárias:

$$
\frac{d S}{d t}=-\beta \frac{I}{N} S, \quad \frac{d I}{d t}=\beta \frac{I}{N} S-\gamma I \text { e } \frac{d R}{d t}=\gamma I \text {. }
$$

Tais equações podem ser simplificadas, conforme Bansal, Grenfell e Meyers [4]:

$$
\begin{aligned}
& \frac{d S}{d t}=-\lambda S \\
& \frac{d I}{d t}=\lambda S-\gamma I \\
& \frac{d R}{d t}=\gamma I
\end{aligned}
$$

Nas equações anteriores $\lambda$ é a taxa na qual os indivíduos estão suscetíveis a ficarem infectados (i.e. "a força da infecção") [3] e $\gamma$ é a taxa de recuperação para indivíduos infectados. Ainda Devemos considerar também as seguintes hipóteses sobre o modelo:

- todos os indivíduos nascem suscetíveis;

- infectados que se recuperam, ganham imunidade total;

- as interações entre os componentes se dão de forma homogênea;

- o tamanho da população permanece constante em relação ao tempo;

- não consideramos emigração ou imigração;

- não consideramos vacinação (campanha de imunização). 
$\mathrm{Na}$ literatura científica há variações deste modelo quando se considera outros fatores, como apresentado na próxima subseção.

\subsection{Modelo SEIRD}

No modelo SEIRD, dividimos a população em 5 grupos: suscetível, exposto, infectado, recuperado e morto. Os parâmetros são similares daqueles do modelo SRI. A população exposta difere da população infectada sobre o desenvolvimento de sintomas; um indivíduo com o vírus entra primeiro no grupo exposto, carregando o vírus durante o período de incubação; então, com o desenvolvimento dos sintomas, o indivíduo passa para o grupo infectado.O fluxo do presente modelo pode ser considerado da seguinte forma:

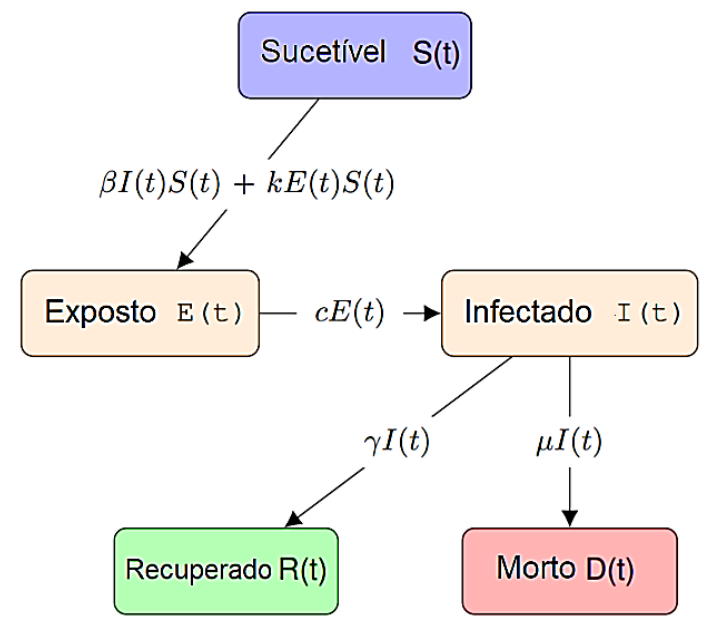

Figura 1: Adaptado de Cintra e Fontinele [4]

A equações diferenciais que descrevem o modelo são:

$$
\begin{aligned}
& \frac{d S}{d t}=-\frac{\beta}{N} I(t) S(t)-\frac{k}{N} E(t) S(t) \\
& \frac{d E}{d t}=\frac{\beta}{N} I(t) S(t)+\frac{k}{N} E(t) S(t)-c E(t) \\
& \frac{d I}{d t}=c E(t)-\gamma I(t)-\mu I(t) \\
& \frac{d R}{d t}=\gamma I(t) \\
& \frac{d D}{d t}=\mu I(t)
\end{aligned}
$$

A taxa de recuperação é representada por $\gamma$ e a taxa de mortalidade $\mu$ são representadas em termos da taxa de mortalidade por casos (CFR) $P_{: \text {: }}$ e o tempo médio desde o início dos sintomas até a recuperação $\tau_{r}$ e morte $\tau_{d}$.

$$
\begin{gathered}
\mu=\frac{P_{:(}}{\tau_{d}} \\
\gamma=\frac{1-P_{:(}}{\tau_{r}}
\end{gathered}
$$

Todas as equações consideram que a população total $N$ se conserva no tempo e é homogênea por simplicidade [4].

\subsection{Modelo de Física do Estado Sólido como Modelo de Previsão}

Uma das curvas mais conhecida é a curva "sigmóide" amplamente utilizada em Ciências e Engenharia de Materiais, Computação, Economia e Sistemas Ecológicos. O nome "sigmóide" vem da forma em $S$ do seu gráfico. Especificamente, em processos de transformação de materiais sólidos, temos a equação de Avrami, algumas vezes chamada de equação de Johnson-Mehl-Avrami-Kolmogorov (JMAK) [5]. A expressão de JMAK estabelece que a cristalinidade desenvolvida por um material (cerâmica, fármacos, metais, polímeros) [6-7] aquecido em regime isotérmico, por um tempo $t$, pode ser correlacionada com o tipo e a cinética de nucleação e crescimento cristalino. Assim, quando as suposições da teoria JMAK são satisfeitas, isto é, quando amostra é infinitamente grande, a nucleação espacialmente é randômica e as taxas de nucleação e crescimento são independentes do tempo, temos

$$
f(t)=1-\exp \left(-k t^{n}\right)
$$

onde $f(t)$ é a fração de massa cristalizada no tempo $t, k$ é a constante cinética e $n$ é o expoente de Avrami, real e positivo. Para obtenção dos parâmetros $n$ e $k$, devemos linearizar a Eq. (1) como se segue:

$$
\begin{aligned}
& 1-f(t)=\exp \left(-k t^{n}\right), \quad \text { passando a ser, } \\
& -\ln (1-f(t))=\left(-k t^{n}\right) \text { e, na sequência: } \\
& \ln \{-\ln [1-f(t)]\}=n \ln (t)+\ln (k)
\end{aligned}
$$

Verificamos que esta dinâmica de transformação de fase para materiais se assemelha a dinâmica de uma epidemia (e neste caso, a pandemia por Coronavírus) [5]. Assim, vamos assumir que o parâmetro $k$ representa a taxa de nucleação e de crescimento de casos. O tempo $t=0$ corresponde à primeira confirmação da doença pelas autoridades de saúde, e, portanto, considerado como o início da epidemia. Para tal análise, devemos construir um gráfico com número de casos que evoluíram no tempo 
$\ln \{-\ln (1-f(t))\}$ versus $\ln (t)$. Para esta figura comparamos a eq. (2) com uma função linear:

$$
\begin{aligned}
\ln \{-\ln [1-f(t)]\} & =n \ln (t)+\ln (k) \\
y & =a x+b
\end{aligned}
$$

A Eq. 13, corresponde a uma função do tipo $y=a x+b$, em que $b=n$ e $a=\ln (k)$. Em seguida, usamos estes parâmetros de ajuste para encontrar $o$ número de casos totais e número diário de casos.

\subsection{Modelo SEIAHR}

O Método SEIAHR, que tem como significado Susceptible, Exposed, Infected, Asymptomatic, Hospitalized, Rehabilitated. Em português: Suscetíveis, Expostos, Infectados, Assintomáticos, hospitalizados e Reabilitados e, é responsável por justamente realizar uma modelagem da evolução temporal entre estas variáveis. Com as considerações realizadas em Rocha Filho et al. [8] passamos a ter neste modelo para o SARS-CoV-2: Indivíduos Susceptíveis $\left(\mathrm{S}_{\mathrm{i}}\right)$, Hospitalizados $\left(\mathrm{H}_{\mathrm{i}}\right)$ por Covid-19, Expostos $\left(\mathrm{E}_{\mathrm{i}}\right)$ (indivíduos em período de incubação viral e que não estão transmitindo a infecção), Infecciosos $\left(\mathrm{I}_{\mathrm{i}}\right)$ e Recuperados $\left(\mathrm{R}_{\mathrm{i}}\right), i=1, \ldots, \mathrm{M}$. Para o $t=0$, temos $\sum_{i=1}^{M} n_{i}=1$; em que a população é representada por um determinado grupo de faixa etária de idade $n_{i}=S_{i}+H_{i}+E_{i}+I_{i}+R_{i} \quad$ e variável $\mathrm{M}$, corresponde ao número de grupos por faixa etária de idade (0-9, 10-39, 40-49, 50-59, 60-69, $\geq 70$ anos).

A probabilidade de transmissão é então obtida realizando alguns ajustes, levando em conta, de forma básica a quantidade do número de interações pessoais

$$
\begin{gathered}
R_{0}=\sum_{i, j=1}^{M} n_{j} \beta_{i j} / \gamma, \text { ou: } \\
R_{0}=\sum_{i, j=1}^{M} n_{j} P_{c} C_{i j} / \gamma
\end{gathered}
$$

É suposto também, para a Eq. 14, que somente os casos graves e críticos sejam hospitalizados [8]. No viés de que os enfermos que se encontram hospitalizados estão na condição de não contato com as pessoas externas ao ambiente hospitalar, obviamente, eles não contribuirão para a força de infecção, obtida da seguinte forma:

$$
\lambda_{i}=\sum_{i=1}^{M} B_{i, j} \frac{I_{j}}{n_{i}}
$$

Assim, o sistema de equações diferenciais que representam o modelo são as seguintes:

$$
\begin{aligned}
& \frac{d S_{i}}{d t}=\kappa \delta_{i, 1}-\lambda_{i} S_{i}-\mu S_{i}+v_{i} S_{i}+v_{i-1} S_{i-1} \\
& \frac{d H_{i}}{d t}=-\psi H_{i}+\zeta_{i} \lambda_{i} E_{i}\left(t-\tau_{1}\right)-\theta \zeta_{i} \lambda_{i} E_{i}\left(t-\tau_{2}\right)-\mu H_{i} \\
& \frac{d E_{i}}{d t}=\lambda S_{i}-\sigma E_{i}-\zeta_{i} \sigma E_{i}-\mu E_{i}-v_{i} E_{i}+v_{i-1} E_{i-1}
\end{aligned}
$$

$$
\frac{d I_{i}}{d t}=\sigma E_{i}-\gamma I_{i}-\mu I_{i}-v_{i} I_{i}+v_{i-1} I_{i-1}
$$

$$
\frac{d R_{i}}{d t}=\gamma I_{i}-\psi H_{i}-\mu R_{i}-v_{i} R_{i}+v_{i-1} R_{i-1}
$$

Para todas as equações diferenciais apresentadas, a dependência das variáveis é em função do tempo, com exceção a Eq. 16, com a presença do delta de Kronecker $\left(\delta_{i, 1}\right)$, em que assume o valor 1 (se $i=j$ ) e, caso ao contrário, o valor será 0) [8]. Apresentamos na Figura 2, o modelo esquemático para a Eq. 20, cuja taxa de transmissão é dada pela Eq. (14) e a força de infecção, é a obtida pela Eq. (15):

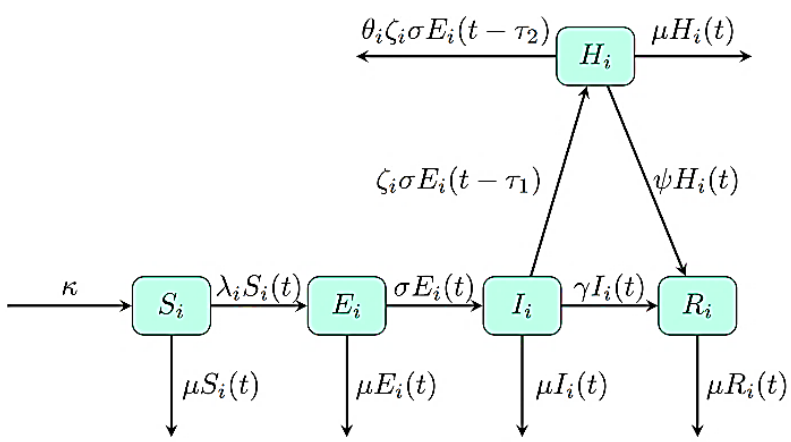

Figura 2: Adaptado de Rocha Filho [8]

\subsection{Modelos preditivos baseados em Gaussianas}

A distribuição de probabilidades normal ou Gaussiana de uma $x$, que segue uma distribuição de probabilidades chamada de normal ou gaussiana tem sua função densidade de probabilidades obtida da seguinte maneira:

$$
f_{x}(x)=\frac{1}{\sqrt{2 \pi} \sigma_{x}} \exp \left[-\frac{1}{2}\left(\frac{x-\mu_{x}}{\sigma_{x}}\right)^{2}\right]
$$

A Eq. 21 depende da média e do desvio padrão da variável $x$, ou seja, $N\left(\mu_{x}, \sigma_{x}\right)$ [9]. A 
distribuição Normal Padrão, é dada pela seguinte relação [9]:

$$
f_{y}(y)=\frac{1}{\sqrt{2 \pi}} \exp \left[-\frac{1}{2} y^{2}\right]
$$

Além das Eq. 21 e Eq. 22, destacamos que a Estatística de Ordem possibilita encontrarmos da distribuição de valores extremos de interesse por meio de uma distribuição inicial da variável aleatória, considerando hipóteses que possam simplificar o modelo [10]. Assim, destacamos as formas assintóticas de convergência, que dependem do decaimento da calda de distribuição inicial, na direção do extremo de interesse. Neste viés, as distribuições estatísticas mais utilizadas são a de Gumbel, Fréchet ou de Weibull [10].

O comportamento realizado pelo Coronavírus, em boa medida, assim como nos modelos preditivos apresentados anteriormente, também pode ser aproximado, considerando um ajuste não-linear por meio do exposto nesta sessão, das Gaussianas.

\section{Discussão dos resultados}

Por meio das principais metodologias apresentadas na sessão anterior, apresentamos na sequência, alguns resultados já estimados em relação à Pandemia de Coronavírus. Nas Figura 3, apresentamos algumas estimativas para a Itália:

Corrigido pela porcentagem de infectados em realação a qte da população

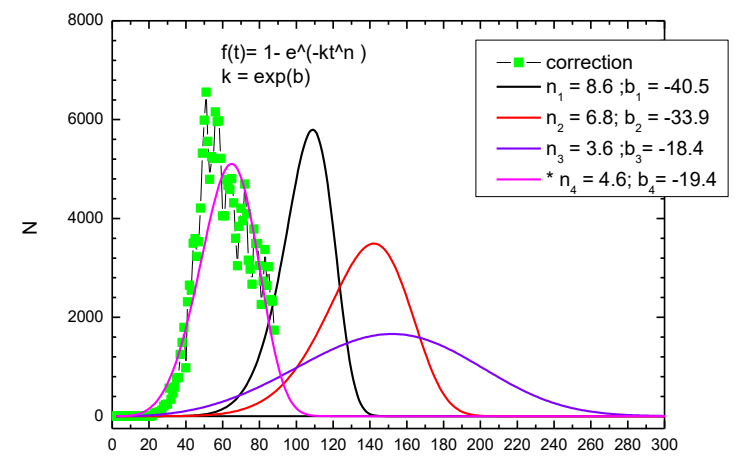

Figura 3: Estimativa realizada por meio da equação de Avrami (JMAK), para a Itália (realizado em 27/04/2020). Fonte: Dos autores.

Na Figura 4, algumas estimativas realizadas para o Brasil, observando os dados fornecidos pelo Ministério da Saúde, até o final de Maio/2020:

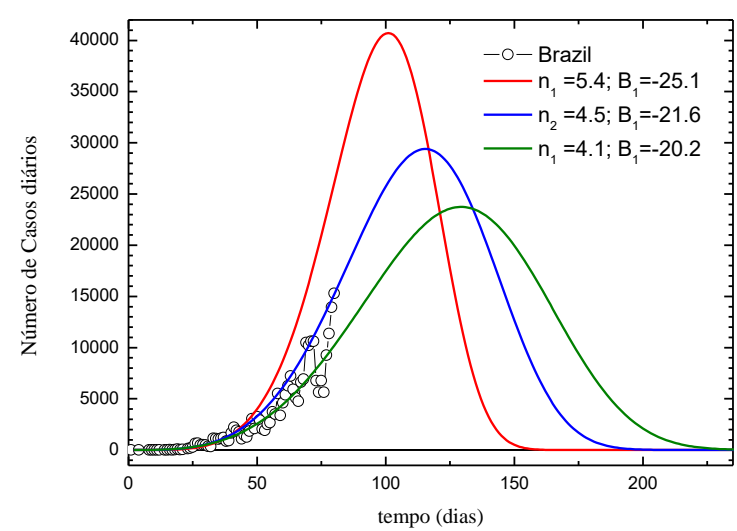

Figura 4: Estimativas realizadas por meio da equação de Avrami (JMAK), para o Brasil (realizado final de maio/2020). Fonte: Dos autores.

$\mathrm{Na}$ Figura 5, apresentamos algumas estimativas para o Estado do Espírito Santo/ES, com os dados fornecidos pelo Ministério da Saúde, na primeira quinzena de Maio/2020.

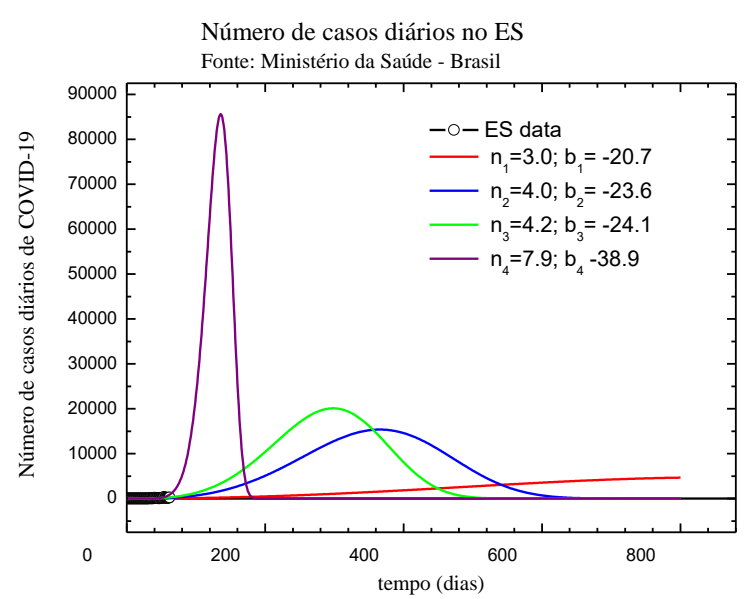

Figura 5: Estimativas realizadas por meio da equação de Avrami (JMAK), para o Estado do Espírito Santo/ES (realizado na primeira quinzena de maio/2020). Fonte: Dos autores.

$\mathrm{Na}$ Figura 6, apresentamos algumas estimativas para o Estado do Pará, por meio do modelo preditivo baseado na equação de Avrami:

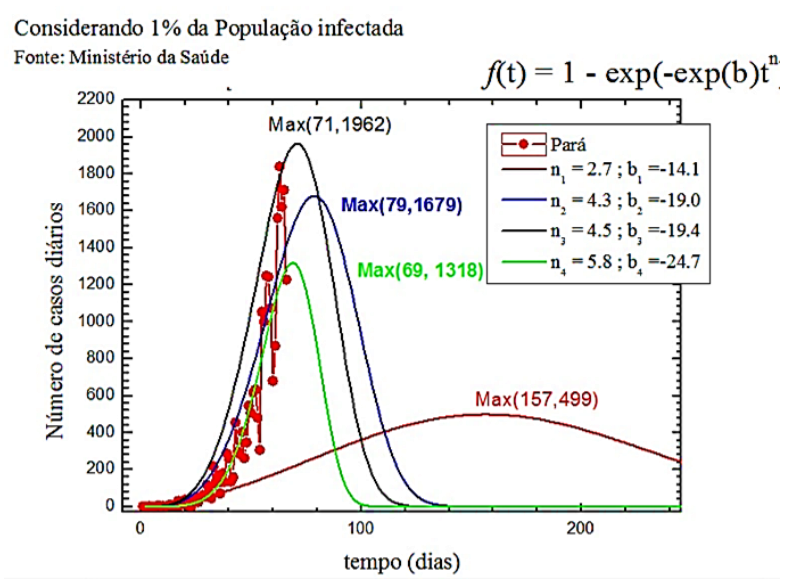


Figura 6: Estimativas realizadas por meio da equação de Avrami (JMAK), para o Estado do Pará/PA (realizado na primeira quinzena de junho/2020). Fonte: Dos autores.

Na Figura 7, utilizamos modelos preditivos baseados em Gaussianas, para o Estado do Pará:

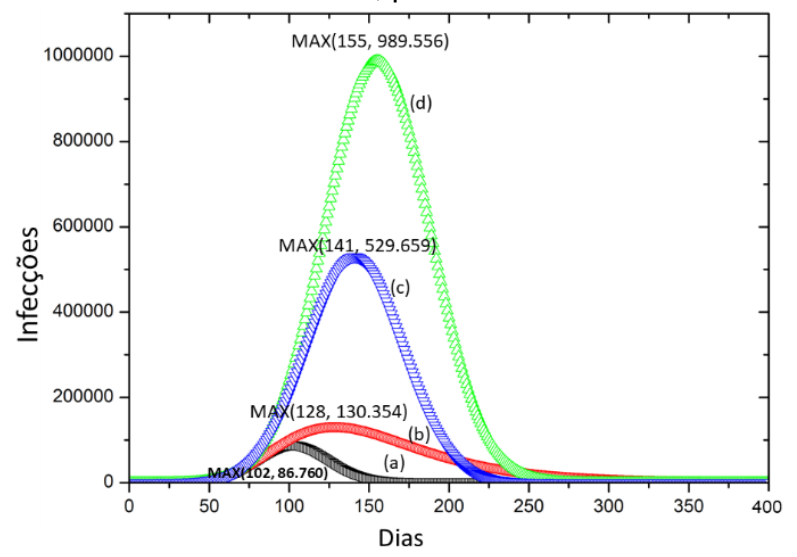

Figura 7: (a) Ajuste não-linear realizado para a curva $y=y_{0}+$ $\left(\mathrm{A} /\left(\mathrm{w}^{*} \operatorname{sqrt}(\mathrm{PI} / 2)\right)\right)^{*} \exp \left(-2^{*}\left(\left(\mathrm{x}-\mathrm{x}_{\mathrm{c}}\right) / \mathrm{w}\right)^{\wedge} 2: \mathrm{y}_{0}=78,68902, \mathrm{x}_{\mathrm{c}}=102,45252, \mathrm{w}\right.$ $=44,59459, A=4,84572 E 6$, com o ajuste $R^{2}=0,99901$;

(b) Ajuste não-linear realizado para a curva $y=y_{0}+A^{*} \exp (-\exp (-z)-z+1)$, $z=\left(x-x_{c}\right) / w: y_{0}=204,64804, x_{c}=128,47821, w=43,76523, A=$ 130157,7524 , com o ajuste $R^{2}=0,99892$;

(c) Ajuste não-linear realizado para a curva $y=\exp \left(a+b^{*} x+c^{*} x^{\wedge} 2\right): a=$ $2,28633, x_{c}=140,9635060, b=0,15456, c=-5,48227 E(-4)$, com o ajuste $R^{2}=0,99933$;

(d) Ajuste não-linear realizado para a curva $y=\mathrm{y}_{0}+$ $\left(\mathrm{A} /\left(\mathrm{w}^{*} \mathrm{sqrt}(\mathrm{Pl} / 2)\right)\right)^{*} \exp \left(-2^{*}((\mathrm{x}-\mathrm{xc}) / \mathrm{w})^{\wedge} 2: \mathrm{y}_{0}=-61,73036, \mathrm{x}_{\mathrm{c}}=155,12796, \mathrm{w}\right.$ $=65,52222, A=8,1268 \mathrm{E} 6$, com o ajuste $R^{2}=0,99935$;

Fonte: Dos autores.

Ressaltamos que, em relação à Figura 7 (cujos cálculos foram realizados na semana final de Junho/2020), atualmente os dados oficiais para o Estado do Pará, ainda se encontram entre as curvas (b) e (c), apenas em estimativa de quantitativo de infectados (grifos nossos).

Nessa semana, em que os ajustes nãolineares para o Estado do Pará foram realizados, os números oficiais apontaram para 100mil infectados. Baseado nas análises realizadas, a curva (d), corresponde à estimativa de números reais de infectados no Pará, significando dizer que: pela falta de testagem realizada na população, em que o número de 100 mil infectados foram os que de fato estiveram diagnosticados por meio de exames, a estimativa em números reais correspondia em $\underline{989.556}$ (aproximadamente 1 milhão) ${ }^{1}$ Paraenses que já tinham tido contado com o Coronavírus ou estavam infectados (Grifos nossos).

Na Figura 8, apresentamos os novos casos de infecções diários e na Figura 9, o total de óbitos por meio do modelo preditivo utilizando o modelo SEIAHR [8] para o Estado do Pará. As variáveis necessárias ao modelo à resolução das equações diferenciais $\psi$,

\footnotetext{
${ }^{1}$ Sugerimos a entrevista disponibilizada em: https://www.youtube.com/watch?v=cJVKpAnmjsg e https://www.youtube.com/watch?v=bxL-1h7WS6s
}

$L^{(0)}, \theta, \sigma, \gamma, \zeta, \tau_{1}, \tau_{2}, \chi$ e $\xi$, apresentam seus valores disponibilizados em ALMEIDA et al. [11], bem como os demais detalhamentos para as predições:

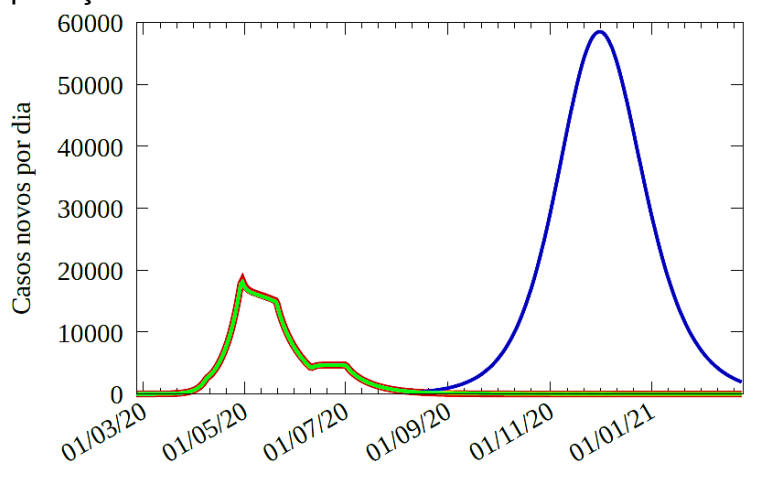

Figura 8: Estimativas realizadas por meio o modelo SEIAHR $[8,11]$, para o Estado do Pará/PA (realizado ao fim da primeira quinzena de agosto/2020). Fonte: adaptado de [11].

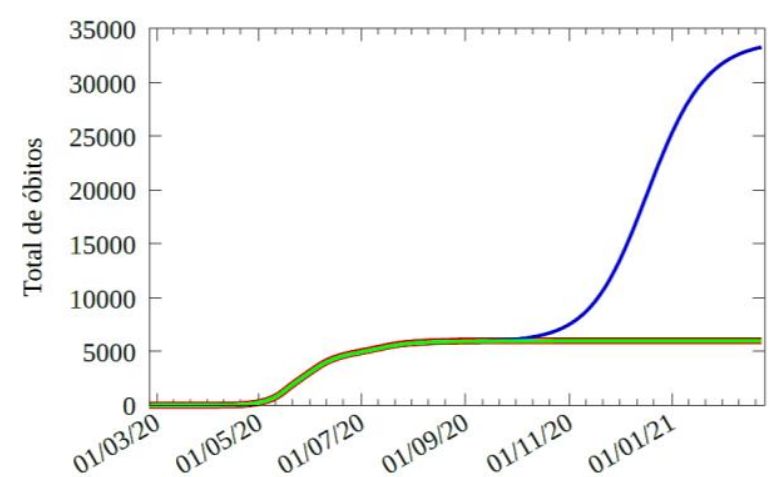

Figura 9: Estimativas realizadas por meio o modelo SEIAHR $[8,11]$, para o Estado do Pará/PA (realizado ao fim da primeira quinzena de agosto/2020). Fonte: adaptado de [11].

Pelos resultados apresentados alicerçados no modelo predito SEIAHR, podemos perceber, em termos de estimativas, que para o final do ano de 2020 e começo do ano de 2021, após as festas (natal e réveillon) para o Pará, não são bons, diante da flexibilização que ocorre/ocorreu no Estado.

As estimativas, ratificam que, a referida flexibilização com a falta de consciências das pessoas, em termos de relaxarem ou até mesmo abandonarem as medidas de profilaxia recomendadas para Organização Mundial de Saúde (OMS) e até mesmo o Ministério da Saúde, consequentemente, realizaram incomensuráveis aglomerações, resultado na já sobrecarga na rede privada de saúde e elevado aumento na procura por atendimento médico na rede pública de saúde, por casos de Covid-19. Há fortes indícios de que possa ocorrer uma segunda onda para o Pará, mais intensa que a primeira, se o atual padrão de comportamento populacional e políticas públicas continuarem! $!^{2}$

\footnotetext{
${ }^{2}$ Indicamos a entrevista internacional disponível em: https://www.youtube.com/watch?v=9 jemV0Kx04
} 
Semelhantemente às Figura 8 e Figura 9. apresentamos nas Figura 10 e Figura 11, os novos casos de infecções diários para o Estado do Espírito Santo/ES e o total de óbitos, respectivamente, utilizando o modelo SEIAHR. Seguindo ainda de forma atenda as observações citadas anteriormente em relação às equações diferenciais, seguem os gráficos com informações estimativas:

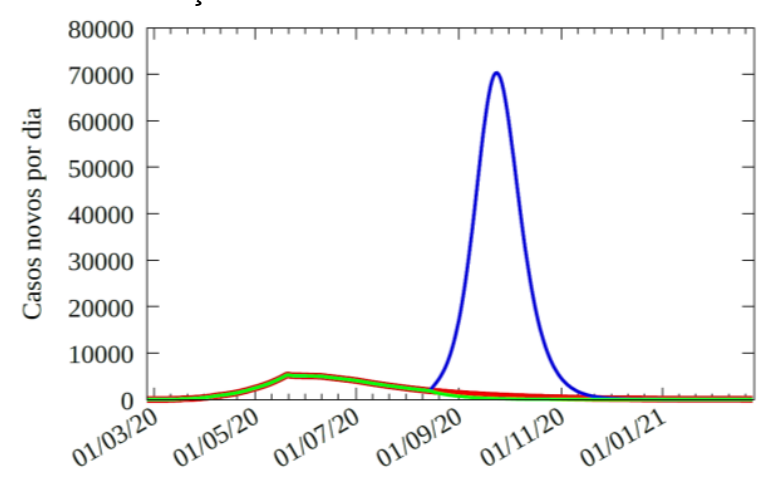

Figura 10: Estimativas realizadas por meio o modelo SEIAHR [8,11], para o Estado do Espírito Santo, para as novas infecções diárias (realizado ao fim da primeira quinzena de agosto/2020). Fonte: adaptado de [11].

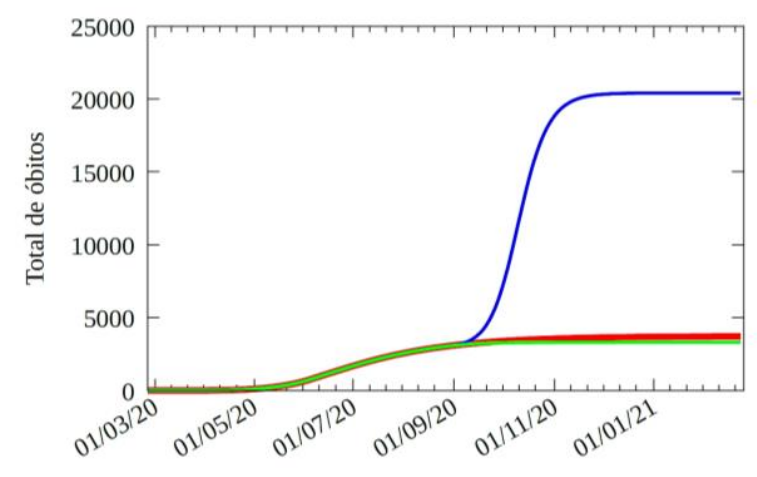

Figura 11: Estimativas realizadas por meio o modelo SEIAHR [8,11], para o Estado do Espírito Santo, considerando o total de óbitos (realizado ao fim da primeira quinzena de agosto/2020). Fonte: adaptado de [11].

\section{Considerações finais}

Como dito durante este artigo, nosso objetivo aqui foi o de apresentar cenários epidemiológicos à difusão do Coronavírus. Indicamos/sugerimos 5 metodologias diferentes para que pudéssemos realizar estimativas em relação à difusão do SARS-CoV-2. Longe de nossas pretensões limitarmos o quantitativo de modelos de previsão à utilização em estimativas epidêmicas. Contudo, dos diversos modelos epidemiológicos presentes na literatura científica, ratificamos que por uma questão de escolha dos autores, apresentamos aqui o modelo SIR, o modelo SEIRD, o modelo de Física do Estado Sólido (Avrami ou JMAK), o modelo SEIAHR e os ajuste baseados em Gaussianas.

Dos métodos que apresentamos, escolhemos também para apresentação dos resultados: a equação de Avrami ou JMAK, as Gaussianas e o modelo SEIAHR. Com os resultados parciais apresentados aqui, apresentamos a necessidade de realizarmos análises dos dados confiáveis para dar suporte as políticas públicas eficientes para o combate à Pandemia. Desta forma, nossa expectativa foi de forma suscinta, colaborar com a compreensão do quadro epidemiológico por meio de cenários epidemiológicos testados por modelos matemáticos que descrevem a difusão da doença infecciosa de transmissão pessoa a pessoa. E predizer quais parâmetros variam em cada cenário.

\section{Agradecimentos}

Jefferson Nascimento agradece a oportunidade de ter realizado pós-doutoramento no departamento de modelagem computacional no CIMATEC/BA (em Sistemas Complexos - Cenários Epidemiológicos cujo foco foi a difusão do Coronavírus no Brasil). Marcelo A. Moret agradece ao $\mathrm{CNPq}$ pelo suporte financeiro parcial oriundo de sua bolsa de Produtividade em Pesquisa (No. 305291/2018-1).

\section{Referências}

[1] NASCIMENTO, J. O. do. Relatório técnico de atividades de pós-doutorado. Salvador/BA: Sistemas Complexos - Cenários Epidemiológicos, 2020. Relatório (Pós-doutorado) - Departamento/Programa de Modelagem Computacional, CIMATEC, 2020.

[2] KERMACK, W. O.; MCKENDRICK, A. G. 1991 Contributions to the mathematical theory of epidemics I-1927. Bull. Math. Biol. 53, 33-55.

[3] SAVI, P V.; SAVI, M. A.; BORGES, B. A mathematical description of the dynamics of coronavirus disease 2019 (COVID-19): A case study of Brazil. (Preprint). Disponível em <http://arxiv.org/abs/2004.03495>. Acesso em: 15 Nov. 2020.

[4] BANSAL, S.; GRENFELL, B.T.; MEYERS, L. A. When individual behaviour matters: homogeneous and network models in epidemiology, J. R. Soc. Interface 4 (16) (2007) 879-891.

[4] Estimative of real number of infections by COVID-19 on Brazil and possible scenarios. CINTRA, H. P. C; FONTINELE, F. N. The preprint server for Health Sciences, 2020 (Preprint). Disponível em <https://doi.org/10.1101/2020.05.03.20052779>. Acesso em: 11 Nov. 2020.

[5] PASSOS, C. A. C.; VIEIRA, E. A.; LOURENCO, J. A.; NASCIMENTO, J. O. do. Characteristics and evolution of COVID-19 cases in Brazil: mathematical modeling and simulation. medRxiv - the preprint server for Health Sciences, 2020 (Preprint). Disponível em < https://www.medrxiv.org/content/10.1101/2020.10.14.20 212829v1>. Acesso em: 09 Nov. 2020. doi: https://doi.org/10.1101/2020.10.14.20212829. 
[6] SHI, J.; HE, F.; XIE, J., LIU, X.; YANG, H. Kinetic analysis of crystallization in Li2O-Al2O3-SiO2-B2O3$\mathrm{BaO}$ glass-ceramics, Journal of Non-Crystalline Solids, Volume 491, 2018, Pages 106-113, ISSN 0022-3093, doi.org/10.1016/j.jnoncrysol.2018.04.004.

[7] BRITO, A. C.; VINHAS, G. M.; ALMEIDA, Y. M. B. de, Wellen, M. R. W.; Canedo, E. L.; Rabello, M. S. (2012). Efeito da adição de PHB na cristalização a frio do PET. Polímeros, 22(2), 111-116. doi.org/10.1590/S0104-14282012005000016

[8] ROCHA FILHO, T. M.; SANTOS, F. S. G. dos; GOMES, V. B.; ROCHA, T. A. H.; CRODA, J. H. R.; RAMALHO, W. M.; ARAÚJO, W. N. de. Expected impact of COVID-19 outbreak in a major metropolitan area in Brazil. The preprint server for Health Sciences, 2020 (Preprint). Disponível em < https://www.medrxiv.org/content/10.1101/2020.10.14.20 212829v1>. Acesso em: 12 Nov. 2020. doi: https://doi.org/10.1101/2020.10.14.20212829.

[9] IBARRA, M. A. C. Análise comparativa de métodos para estimativa de valores extremos de processos aleatórios não-gaussianos. 106f. Dissertação (Mestrado - Programa de Engenharia Civil) - UFRJ / COPPE /, 2017.

[10] ANG, A.H.S., \& TANG, W.H., (1984). "Probability Concepts in Engineering, Planning and Design”, Vol. II, John Willey and Sons, New York, USA.

[11] ALMEIDA, A.C.G.; CORDEIRO, A. J. A.; SCORZA, F. A.; MORET, M. A.; ROCHA FILHO, T. M.; RAMALHO, W. M. Nota Técnica (13/08/2020) Situação da pandemia de Covid-19 no Brasil. UNB, Brasília, DF, 2020. 